\title{
Accuracy of Three-Dimensional Planning in Surgery-First Orthognathic Surgery: Planning Versus Outcome
}

\author{
Ngoc Hieu Tran ${ }^{\mathrm{a}}$, Syrina Tantidhnazet ${ }^{\mathrm{b}, \mathrm{c}}$, Somchart Raocharernporn ${ }^{\mathrm{a}}$, \\ Sirichai Kiattavornchareon ${ }^{\mathrm{a}}$, Verasak Pairuchvej, \\ Natthamet Wongsirichat ${ }^{\mathrm{a}, \mathrm{c}}$
}

\begin{abstract}
Background: The benefit of computer-assisted planning in orthognathic surgery (OGS) has been extensively documented over the last decade. This study aimed to evaluate the accuracy of three-dimensional (3D) virtual planning in surgery-first OGS.

Methods: Fifteen patients with skeletal class III malocclusion who underwent bimaxillary OGS with surgery-first approach were included. A composite skull model was reconstructed using data from conebeam computed tomography and stereolithography from a scanned dental cast. Surgical procedures were simulated using Simplant O\&O software, and the virtual plan was transferred to the operation room using 3D-printed splints. Differences of the 3D measurements between the virtual plan and postoperative results were evaluated, and the accuracy was reported using root mean square deviation (RMSD) and the Bland-Altman method.
\end{abstract}

Results: The virtual planning was successfully transferred to surgery. The overall mean linear difference was $0.88 \mathrm{~mm}(0.79 \mathrm{~mm}$ for the maxilla and $1 \mathrm{~mm}$ for the mandible), and the overall mean angular difference was $1.16^{\circ}$. The RMSD ranged from 0.86 to $1.46 \mathrm{~mm}$ and $1.27^{\circ}$ to $1.45^{\circ}$, within the acceptable clinical criteria.

Conclusion: In this study, virtual surgical planning and 3D-printed surgical splints facilitated the diagnosis and treatment planning, and offered an accurate outcome in surgery-first OGS.

Keywords: CAD/CAM splints; Orthognathic surgery; Surgery-first approach; Virtual planning

Manuscript submitted February 4, 2018, accepted February 20, 2018

${ }^{a}$ Department of Oral and Maxillofacial Surgery, Faculty of Dentistry, Mahidol University, 6 Yothi Street Rachathewee District, Bangkok 10400, Thailand

${ }^{b}$ Department of Orthodontics, Faculty of Dentistry, Mahidol University, 6 Yothi Street Rachathewee District, Bangkok 10400, Thailand; Natthamet Wongsirichat, Department of Oral and Maxillofacial Surgery, Faculty of Dentistry, Mahidol University, 6 Yothi Street Rachathewee District, Bangkok 10400, Thailand. Email: natthamet.won@mahidol.ac.th

${ }^{\mathrm{c} C o r r e s p o n d i n g ~ A u t h o r: ~ S y r i n a ~ T a n t i d h n a z e t, ~ D e p a r t m e n t ~ o f ~ O r t h o d o n t i c s, ~}$ Faculty of Dentistry, Mahidol University, 6 Yothi Street Rachathewee District, Bangkok 10400, Thailand. Email: syrina.tan@mahidol.ac.th

doi: https://doi.org/10.14740/jocmr3372w

\section{Introduction}

Orthognathic surgery (OGS) is a common procedure to treat dentofacial deformities. In the 1960s, surgeons rarely depended on orthodontic treatment for teeth movement prior to surgery. They performed OGS either before orthodontic treatment or after removing orthodontic appliances. Subsequently, the three stages of conventional surgical orthodontic treatment became popular because of the stability of the results and the satisfaction of post-treatment outcomes. In recent years, there has been a growing interest in the surgery-first approach (SFA) OGS [14] since it was first suggested in 1973 by Bell and Creekmore [5]. Epker and Fish [6] explicated the advantages of SFA, which include early improvement of facial form and function, rapid orthodontic tooth movement, and increased stability. Moreover, better patient compliance was observed, due to the initial correction of facial esthetics. Indications and guidelines for SFA were further suggested by Liou et al in 2011 [7].

Conventional treatment planning for OGS involves diagnosis with two-dimensional (2D) cephalometric radiography, face-bow transfer, model surgery on plaster dental cast, and fabrication of intermediate and final occlusal splints. This method remains the most widespread and standard procedure to achieve surgical planning in OGS. The success of two-jaw surgery depends on surgical technique and precise surgical planning $[8,9]$ and the outcome is generally satisfactory. However, 2D tools are conventionally used to attempt at prediction of three-dimensional (3D) surgical and orthodontic movements. Moreover, a final 3D visual treatment objective is not provided to further guide surgical and orthodontic precision because of inherent limitations, such as procedural delay, complexity, and inaccuracy $[9,10]$.

Computer-aided surgical simulation utilizing 3D images from multi-slice computed tomography/cone beam computed tomography (CBCT) has been successfully performed to plan craniofacial surgery. Virtual surgical planning, combined with a method of transferring the plan to surgery, permits maxillofacial surgeons to make an accurate diagnosis, provides a predictable means for $3 \mathrm{D}$ reconstruction, and facilitates the analysis of postoperative changes in both hard and soft tissues. Several articles have described 3D virtual surgical planning protocols [9, 11-13]. However, there are only a few literatures that have investigated clinical feasibility of $3 \mathrm{D}$ planning in SFA [14-17], most of them reporting one or two cases.

The purpose of this study was to evaluate the accuracy of 

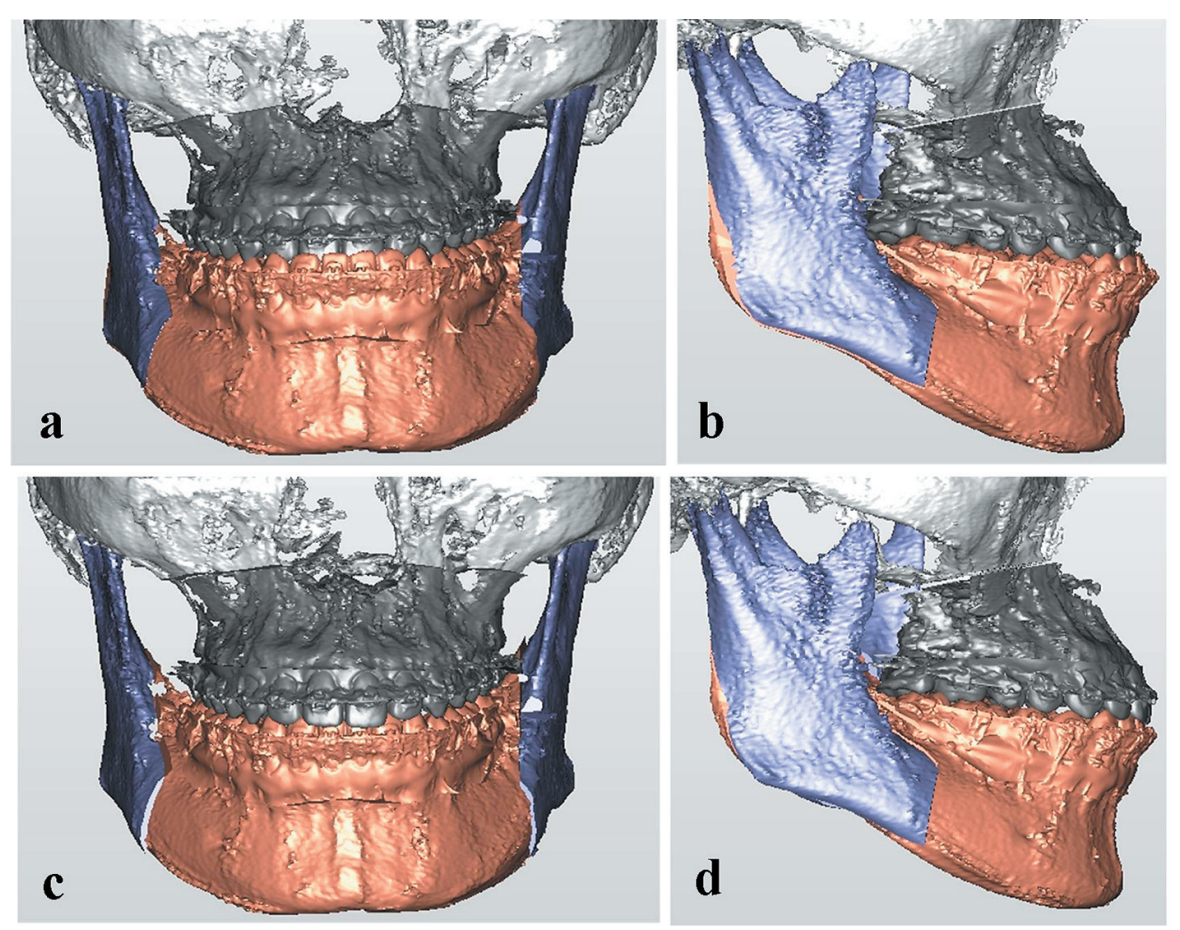

Figure 1. Virtual surgical planning of a 25-year-old male with skeletal Class III malocclusion, retrognathic maxilla, prognathic mandible. (a, b) Preoperative: cutting lines of Le Fort I osteotomy and BSSO were designed. (c, d) Planned postoperative reposition of maxillary and mandibular distal segments.

virtual surgical planning, in comparison with actual outcomes in a series of 15 patients.

\section{Materials and Methods}

\section{Patients}

Fifteen patients who required OGS at Faculty of Dentistry, Mahidol University (Bangkok, Thailand) from December 2015 to July 2017 were recruited for this study. The study was approved by Mahidol University Institutional Review Board (MU-IRB) with COA.No.MU-DT/PY-IRB 2016/037.2408 and all participants signed an informed consent agreement. The study followed the guidelines of the Declaration of Helsinki.

The inclusion criteria were: 1) skeletal class III dentofacial deformities; 2) indication for bimaxillary surgery-first OGS; and 3) high quality CBCT images before $\left(T_{o}\right)$ and 1 month $\left(T_{1}\right)$ after OGS.

The exclusion criteria were: 1) history of craniofacial trauma or OGS; and 2) active temporomandibular joints disorders.

All surgical procedures were performed by one experienced surgeon.

\section{Virtual surgical planning}

High resolution CBCT (Kodak 9500 Cone Beam 3D System, Carestream Dental LLC, Atlanta, GA) taken before the surgery was imported into the planning software Simplant O\&O (Materialise Dental NV, Leuven, Belgium). A 3D virtual model was generated and a surface scan of the plaster model (R900 3D Scanner, 3Shape, Copenhagen, Denmark) was fused to the virtual model. The virtual head orientation was based on a clinical evaluation and an orthodontist's modification: 1) upright position of the patient without tilting the head; 2) clinical findings or facial photographs are correlated with the $3 \mathrm{D}$ constructed skull image; and 3) the FHP of the 3D skull is oriented parallel to the floor. Stereolithographic files of the surface scans of dental plaster models of both arches were fused with the previously aligned dataset by voxel-based matching methods. Dental models registered and scanned under final occlusal position were imported to the surgical plan as a template of the final position of mandible. After a comprehensive 3D evaluation, virtual surgical planning and simulation were performed by the responsible surgeon to separate maxilla at Le Fort I level and mandible by bilateral sgittal split osteotomy (BSSO) at the ascending rami. As a result, five independent segments (midface, maxilla, distal segment of mandible, left and right ramus) of the patient's skull were generated (Fig. 1). The treatment plan was sent to the $\mathrm{CAD} / \mathrm{CAM}$ center and two surgical splints were fabricated by means of rapid-prototyping. The first splint would guide the repositioning of segmented maxilla and the second would decide the final position of the mandible.

\section{Surgical technique}

The surgeries were performed by a single surgeon, with dental 

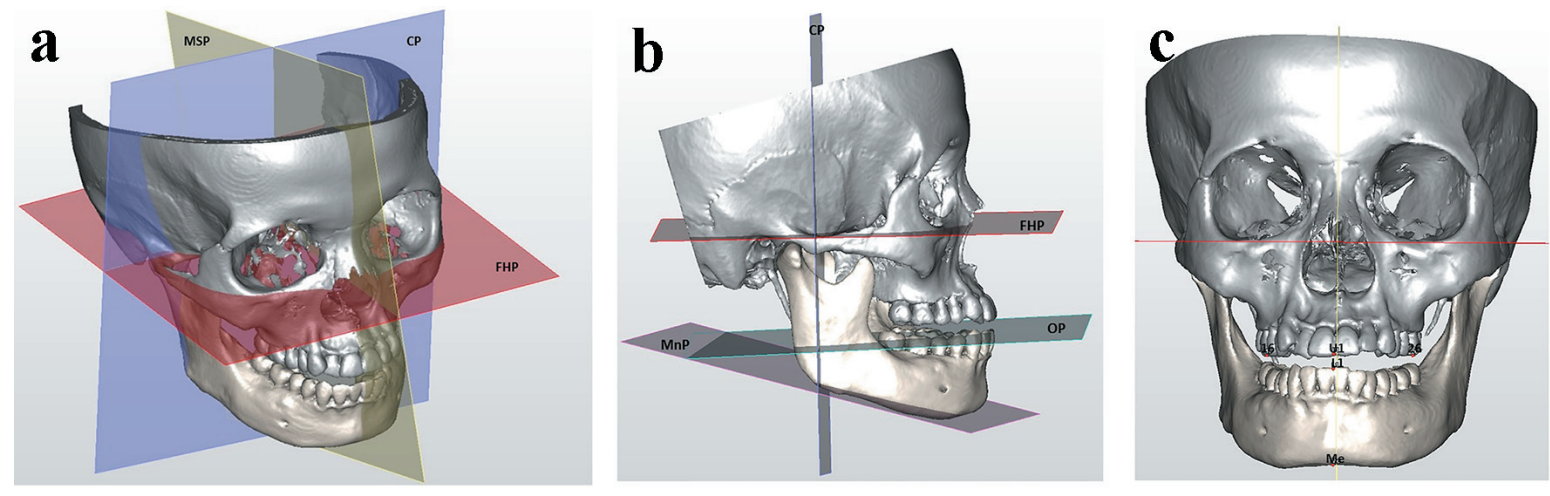

Figure 2. Symmetry planes and landmarks on the surface of the skull. (a) FHP: Frankfort horizontal plane; MSP: midsagittal plane; CP: coronal plane. (b) OP: occlusal plane; MnP: mandibular plane. (c) U1: midpoint of the contact of the upper central incisors; L1: midpoint of the contact of the lower central incisors; 16 and 26: mesiobuccal cusp tips of the upper first molars; Me: menton.

occlusion guidance by CAD/CAM surgical splints. A bonding procedure for inter-maxillary fixation was performed before OGS. Surgery involved a Le Fort I osteotomy followed by mandibular setback using a BSSO. Rigid internal fixation was used after confirmation of the surgical plan and the final assessment of facial symmetry and profile by the surgeon. Three days after the surgery, the elastics for the maxilla-mandibular fixation were applied and continued up to 3 weeks.

\section{Outcome evaluation}

The postoperative CBCT images of patients were taken within 1 month after OGS and 3D models were constructed. If the dentition was not identifiable for landmark digitalization, then the preoperative dental models were imported to replace the dentition for obtaining a clear virtual postoperative 3D model.

To evaluate the accuracy of virtual planning in two-jaw OGS, symmetry planes and landmarks on the surface of the skull were defined. The Frankfort horizontal plane (FHP, connecting the right orbitale, left orbitale, and middle point of the right and left porions) was used because it is unaffected by most craniofacial anomalies. Corresponding to the FHP, four reference planes were constructed (Fig. 2), including the midsagittal plane (MSP, perpendicular plane to the FHP through the nasion), coronal plane (CP, perpendicular plane to the FHP through the sella point), the occlusal plane (OP, connecting the midpoint of the lower incisors with the mesiobuccal cusps of the mandibular first molars, and the mandibular plane (MnP, connecting the menton with the right and left mandibular angle points). Midpoint of the contact of the maxillary and mandibular central incisors (U1, L1), the mesiobuccal cusps of the maxillary right and left first molars $(16,26)$ and the menton (Me) were the five chosen volumetric landmarks (Fig. 2c). The distance between the selected landmarks and the symmetry planes was measured, and the difference between simulated and postoperative model was calculated. For angular analysis, values of the angles constructed by the occlusal and mandibular planes to the FHP and the MSP, respectively, were determined on simulated and postoperative models, and the differ- ence between the two models was calculated (Table 1). All 3D distance and angular measurements were conducted on both surgical plan and postoperative CBCT by a single investigator, so that the inaccuracy between viewers could be minimized.

\section{Statistical analysis}

All data were analyzed by using SPSS 18.0 (SPSS Inc., Chicago, IL). Kolmogorov-Smirnov and Shapiro-Wilk tests were used to test for normality of differences. Paired $t$-test was used to calculate the difference between the virtual planned position and the actual position of the jaws and teeth. Pearson correlation coefficient was used to evaluate intra-observer reproducibility of the measurements. Statistical significance was set at $\mathrm{P}<0.05$. The accuracy was reported using root mean square deviation (RMSD) and the Bland-Altman method [18] for assessing measurement agreement.

In order to interpret results of accuracy of the surgical plan, a linear difference between surgical plan and postoperative outcome smaller than $2 \mathrm{~mm}$ and angular differences smaller than $4^{\circ}$ was considered clinically insignificant [19-22]. However, for the maxillary dental midline position, the most noticeable parameter, a threshold of $1 \mathrm{~mm}$ was used.

\section{Results}

Fifteen participants with five males and 10 females aged 27 \pm 2.75 (ranged from 20 to 35 years old) were recruited in this study after screening hospital documentation and clinical examination. All patients underwent two-jaw surgery.

The virtual surgical planning was successfully transferred to operation room. All the patients were satisfied with the results, including facial profile and occlusion.

The results of the linear and angular measurement differences $(\Delta T)$ between the planning $\left(T_{0}\right)$ and the postoperative result $\left(T_{1}\right)$ in relation to various facial reference planes are shown in Table 1. No significant differences were observed. The overall mean linear difference of the distance between U1, 
Table 1. Measurements and Deviation of Actual Surgery From Surgical Simulation in All Three-Dimensional Landmarks

\begin{tabular}{|c|c|c|c|c|}
\hline \multirow{2}{*}{ Variables } & \multicolumn{2}{|c|}{$T_{0}-T_{1}$} & \multirow{2}{*}{$\mathbf{P}$} & \multirow{2}{*}{ RMSD } \\
\hline & Mean & SD & & \\
\hline \multicolumn{5}{|c|}{ Distance to FHP (mm) } \\
\hline U1 & 0.81 & 0.59 & 0.59 & 1.04 \\
\hline $\mathrm{L} 1$ & 0.89 & 0.53 & 0.53 & 1.18 \\
\hline 16 & -0.97 & 0.34 & 0.47 & 1.39 \\
\hline 26 & -1.07 & 0.37 & 0.79 & 1.26 \\
\hline $\mathrm{Me}$ & 1.25 & 0.43 & 0.14 & 1.45 \\
\hline \multicolumn{5}{|c|}{ Distance to MSP (mm) } \\
\hline U1 & -0.23 & 0.33 & 0.58 & 0.86 \\
\hline $\mathrm{L} 1$ & -0.58 & 0.46 & 0.21 & 0.91 \\
\hline 16 & 0.71 & 0.54 & 0.7 & 1.12 \\
\hline 26 & 0.58 & 0.38 & 0.63 & 1.25 \\
\hline $\mathrm{Me}$ & 0.83 & 0.27 & 0.87 & 1.33 \\
\hline \multicolumn{5}{|c|}{ Distance to CP (mm) } \\
\hline U1 & 0.93 & 0.43 & 0.75 & 1.23 \\
\hline L1 & 0.98 & 0.78 & 0.23 & 1.46 \\
\hline 16 & -1.05 & 0.76 & 0.55 & 1.24 \\
\hline 26 & -1.09 & 0.93 & 0.74 & 1.39 \\
\hline $\mathrm{Me}$ & 1.16 & 0.35 & 0.32 & 1.43 \\
\hline \multicolumn{5}{|c|}{ Plane angulation relative to $\mathrm{FHP}\left(^{\circ}\right)$} \\
\hline OP & 1.06 & 0.69 & 0.28 & 1.29 \\
\hline $\mathrm{MnP}$ & 1.41 & 0.65 & 0.08 & 1.45 \\
\hline \multicolumn{5}{|c|}{ Plane angulation relative to $\operatorname{MSP}\left(^{\circ}\right)$} \\
\hline OP & 0.98 & 0.47 & 0.59 & 1.27 \\
\hline $\mathrm{MnP}$ & 1.18 & 0.51 & 0.23 & 1.37 \\
\hline
\end{tabular}

SD: standard deviation. No significant difference was found.

16, 26, L1 and Me to FHP, MSP and CP was $0.88 \mathrm{~mm}$. The mean linear difference for maxillary landmarks (U1, 16, 26 to FHP, MSP and CP) was $0.79 \mathrm{~mm}$. The mean linear difference for mandibular landmarks (L1, Me to FHP, MSP and CP) was $1 \mathrm{~mm}$. The overall mean linear differences for both maxillary and mandibular landmarks relative to FHP, MSP and CP were $1,0.59$ and $1.04 \mathrm{~mm}$, respectively. The overall mean angular difference was $1.16^{\circ}$. The mean angular differences relative to FHP and MSP were 1.23 and $1.08^{\circ}$, respectively.

The RMSD ranged from 0.86 to $1.46 \mathrm{~mm}$ and 1.27 to $1.45^{\circ}$, all within the acceptable clinical criteria.

Table 1 also presents the absolute mean RMSD between the planned and actual outcomes regarding distance from U1, L1 and Me to MSP, and Table 2 shows their 95\% limits of agreement (Bland-Altman upper and lower limits). The result of the RMSD calculation showed that the accuracy of upper and lower dental midline position was $0.86 \mathrm{~mm}(95 \%$ limits of agreement, -0.89 to $0.42 \mathrm{~mm})$ and $0.91 \mathrm{~mm}(95 \%$ limits of agreement, -1.49 to $0.33 \mathrm{~mm})$, respectively. These results are further illustrated by Bland-Altman plots (Fig. 3) and they
Table 2. Accuracy (Bland-Altman Upper and Lower Limits) of Linear Differences to MSP Between the Planned and Postoperative Outcomes

\begin{tabular}{lll}
\hline & \multicolumn{2}{c}{ Linear difference $(\mathbf{m m}), 95 \%$ CI } \\
\cline { 2 - 3 } & Lower limit & Upper limit \\
\hline U1 & $-0.89(-1.21$ to -0.56$)$ & $0.42(0.1$ to 0.74$)$ \\
L1 & $-1.49(-1.93$ to -1.04$)$ & $0.33(-0.12$ to 0.78$)$ \\
\hline
\end{tabular}

could be considered to be in agreement.

A second surgical simulation was repeated, and measurements relative to FHP were recorded. These measurements were then compared with the initial surgical simulation. The analysis showed less than $0.2 \mathrm{~mm}$ and $0.6^{\circ}$ difference between duplicate measurements. There was no statistically significant difference between the first and second surgical simulation, and the data showed an excellent correlation (Table 3 ).

For illustration of the changes in the facial profile and occlusion, a 25-year-old male with skeletal class III malocclusion, occlusal cant, retrognathic maxilla, prognathic mandible was chosen as a representative case (Fig. 4).

\section{Discussion}

During the last 40 years, investigators have placed infrequent emphasis on the SFA in OGS. In 1977, when the orthodontics-first approach was popular, Epker and Fish [6] suggested that for better repositioning of skeletal and dento-osseous segments, the surgical procedure should be performed prior to the orthodontic treatment. This would ensure esthetic results and accomplish tooth movement safely and easily.

The criteria that are suggested for SFA [7] are: wellaligned to mild crowding, flat to mild curve of Spee, normal to mild proclination/retroclination of incisors, minimal transverse discrepancy. This approach is also indicated in cases in which decompensation is needed. "Even though the surgeryfirst technique can be applied to class II as well as class III malocclusions, the majority of cases treated using this approach have been cases with class III malocclusion meeting the above criteria" [7].

Literatures on virtual surgical planning in OGS are already present. However, most of them report one or several cases to assert the hands-on planning [23-25]. Because of the differences in softwares, landmarks and presentation of data, it was almost impossible to perform a meta-analysis [24]. Lin et al [25] reviewed the reports published in the past 10 years on computer-assisted procedures in OGS, including surgical planning, simulation, intraoperative translation of virtual surgery, and postoperative evaluation. They concluded that using computer-assisted technique in OGS produces optimal functional and esthetic outcomes, patient satisfaction, accurate translation of treatment plan, and facilitation of intraoperative manipulation [25].

Different methods of presentation of data on comparison of planned and actual outcome models following two-jaw OGS were reported, such as intraclass correlation coefficient, 

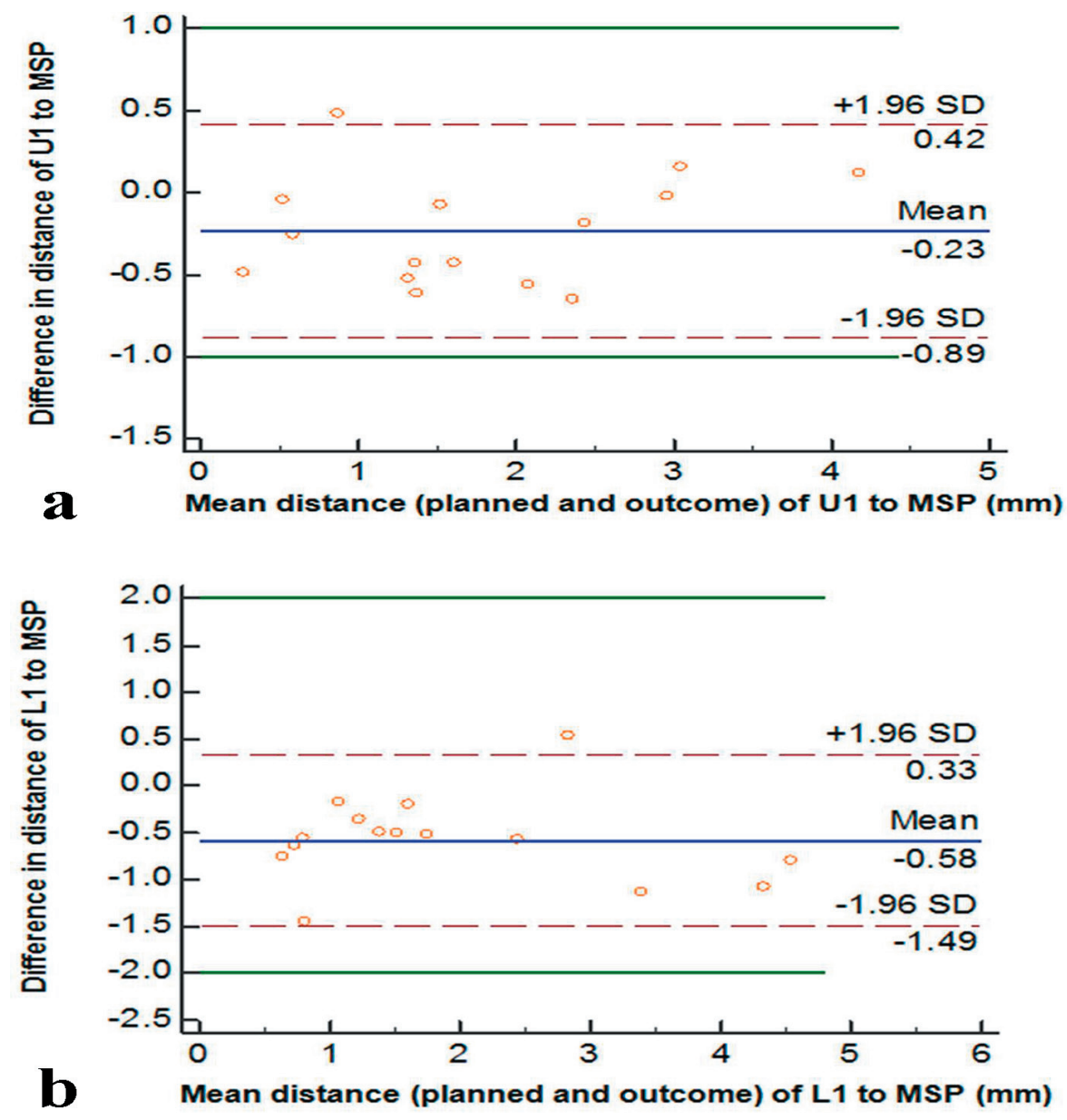

Figure 3. The Bland-Altman plots evaluating variation of difference in measurement between simulation and actual outcome regarding distance from U1 to MSP (a) and L1 to MSP (b). The value is represented as mean \pm 1.96 SDs. Maximum allowed differences were set at $1 \mathrm{~mm}(\mathrm{a})$ and $2 \mathrm{~mm}(\mathrm{~b})$, respectively.

difference of 3D surface area, and linear and angular differences in the dimensions [26-30]. The present study followed three interpretations of outcome assessment: 1) comparison between the measurements of $\mathrm{T}_{0}$ and $\mathrm{T}_{1}$ to explore the variables that differ statistically; 2) RMSD to present the accuracy of the surgery using each measurement variable of the 3D landmarks. Success criteria were set at $2 \mathrm{~mm}$ for the linear

Table 3. Initial Measurements Versus Repeated Surgical Simulations Measurements

\begin{tabular}{lllll}
\hline $\begin{array}{l}\text { First and second } \\
\text { measurements }\end{array}$ & $\mathbf{r}$ & \multicolumn{3}{c}{ Paired differences relative to FHP } \\
\cline { 3 - 5 } Mean & SD & P \\
\hline $\mathrm{U} 1$ & 0.97 & 0.04 & 0.59 & 0.778 \\
$\mathrm{~L} 1$ & 0.98 & 0.19 & 0.69 & 0.3 \\
16 & 0.96 & -0.05 & 0.49 & 0.69 \\
26 & 0.99 & 0.05 & 0.36 & 0.61 \\
$\mathrm{Me}$ & 0.99 & 0.06 & 0.43 & 0.59 \\
$\mathrm{OP}$ & 0.98 & -0.38 & 0.75 & 0.07 \\
$\mathrm{MnP}$ & 0.97 & -0.32 & 0.66 & 0.08 \\
\hline
\end{tabular}

difference, and $4^{\circ}$ for the angular difference in most of the published studies [19-22]. The results showed high accuracy for the chosen landmarks, the outcomes were within clinical threshold, and no significant difference was found. Furthermore, it seems that the maxilla was transferred more precisely than the mandible $(0.79 \mathrm{~mm}$ vs. $1 \mathrm{~mm})$, and the virtual planning provides better control of the deviation from $\operatorname{MSP}(0.59$ $\mathrm{mm})$ than the FHP $(1 \mathrm{~mm})$ and CP $(1.04 \mathrm{~mm})$. For the distance to midline, excellent accuracy of midpoint of upper central incisors was achieved.

The results of mean differences were $0.88 \mathrm{~mm}$ and $1.16^{\circ}$. They were similar to the findings of Zhang et al [29], in which the overall mean linear difference was $0.81 \mathrm{~mm}$, and the overall mean angular difference was $0.95^{\circ}$.

Additionally, the RMSD in this study ranged from 0.86 to $1.46 \mathrm{~mm}$. These results were comparable to the study conducted by Ko et al [30] (1.45 - $2.28 \mathrm{~mm})$ and Hsu et al [26], although the latter utilized the centroid positions of maxilla and mandible.

Combining the SFA with 3D CBCT virtual planning allows us to acquire symmetry in the anatomical structures with great accuracy while significantly increasing treatment man- 

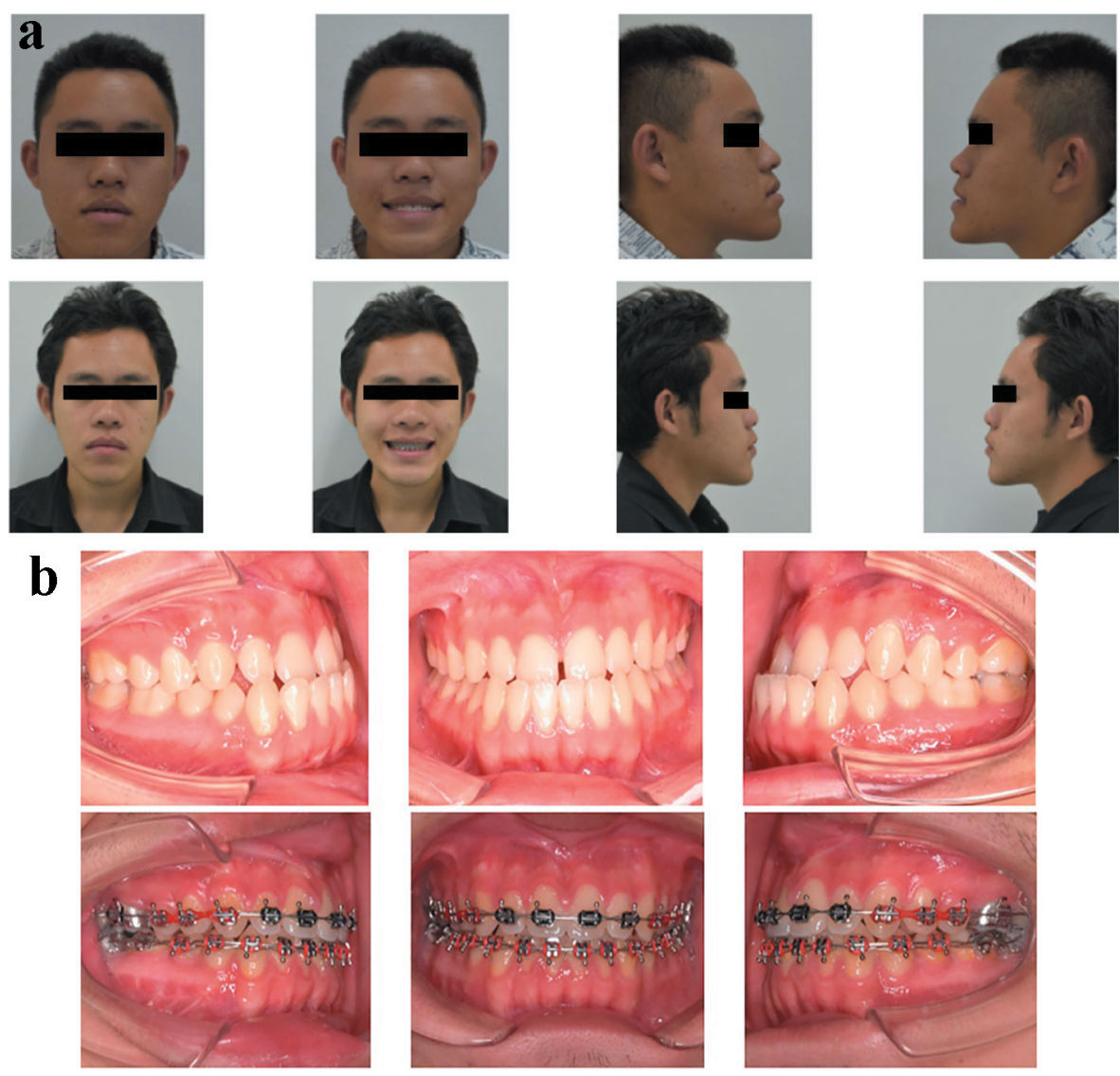

c
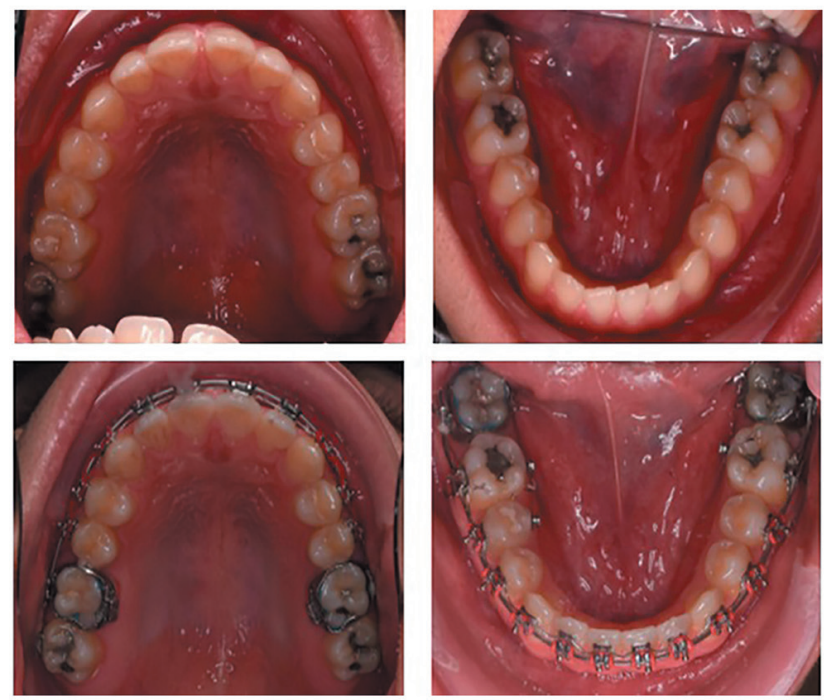

Figure 4. Progress of treatment in the representative patient. (a) Facial profile of the patient before and after surgery. (b, c) Final occlusion of the patient before and after surgery. Pretreatment records showed skeletal class III malocclusion with occlusal cannot and a concave profile due to maxillary retrognathism and mandibular prognathism. Postoperatively, good improvement in the facial profile, leveled maxillary occlusal plane, and good occlusal outcome were achieved.

agement. However, this combination requires a proper team approach between the surgeon and the orthodontist in order to accomplish a precise surgical planning. Using 3D models, all of the procedures for diagnosis and surgical splint production can be simulated, intermediate assessment can be conducted, and the intermediate and final surgical splints can be produced. Therefore, errors encountered during laboratory procedures are minimal. Regarding the advantages of virtual surgical planning, this technique will play an important part in orthognathic surgical procedures in the future. 
In conclusion, fusion of new technologies and techniques such as 3D CBCT-based surgical planning, computer-aided splint fabrication, and the SFA can make OGS more efficient and effective for patients and the surgical-orthodontic team. Accurate and predictable treatment outcomes can be achieved by using these technological advancements.

\section{Acknowledgments}

The authors would like to express our gratitude to the staff, dental assistants and our colleagues in the Department of Oral and Maxillofacial Surgery, Faculty of Dentistry, Mahidol University. We also thank Dr. Bishwa Prakash Bhattarai, for his help in editing.

\section{Funding}

None.

\section{Conflict of Interest}

None.

\section{References}

1. Nagasaka H, Sugawara J, Kawamura H, Nanda R. "Surgery first" skeletal Class III correction using the Skeletal Anchorage System. J Clin Orthod. 2009;43(2):97-105.

2. Sugawara J, Aymach Z, Nagasaka DH, Kawamura H, Nanda R. "Surgery first" orthognathics to correct a skeletal class II malocclusion with an impinging bite. J Clin Orthod. 2010;44(7):429-438.

3. Liao YF, Chiu YT, Huang CS, Ko EW, Chen YR. Presurgical orthodontics versus no presurgical orthodontics: treatment outcome of surgical-orthodontic correction for skeletal class III open bite. Plast Reconstr Surg. 2010;126(6):2074-2083.

4. Villegas C, Uribe F, Sugawara J, Nanda R. Expedited correction of significant dentofacial asymmetry using a "surgery first" approach. J Clin Orthod. 2010;44(2):97-103; quiz 105.

5. Bell WH, Creekmore TD. Surgical-orthodontic correction of mandibular prognathism. Am J Orthod. 1973;63(3):256-270.

6. Epker BN, Fish L. Surgical-orthodontic correction of open-bite deformity. Am J Orthod. 1977;71(3):278-299.

7. Liou EJ, Chen PH, Wang YC, Yu CC, Huang CS, Chen YR. Surgery-first accelerated orthognathic surgery: orthodontic guidelines and setup for model surgery. J Oral Maxillofac Surg. 2011;69(3):771-780.

8. Sharifi A, Jones R, Ayoub A, Moos K, Walker F, Khambay $\mathrm{B}$, McHugh $\mathrm{S}$. How accurate is model planning for orthognathic surgery? Int J Oral Maxillofac Surg. 2008;37(12):1089-1093.
9. Xia JJ, Gateno J, Teichgraeber JF. New clinical protocol to evaluate craniomaxillofacial deformity and plan surgical correction. J Oral Maxillofac Surg. 2009;67(10):20932106.

10. Zizelmann C, Hammer B, Gellrich NC, Schwestka-Polly R, Rana M, Bucher P. An evaluation of face-bow transfer for the planning of orthognathic surgery. J Oral Maxillofac Surg. 2012;70(8):1944-1950.

11. Swennen GR, Mollemans W, Schutyser F. Three-dimensional treatment planning of orthognathic surgery in the era of virtual imaging. J Oral Maxillofac Surg. 2009;67(10):2080-2092.

12. Edwards SP. Computer-assisted craniomaxillofacial surgery. Oral Maxillofac Surg Clin North Am. 2010;22(1):117-134.

13. Bell RB. Computer planning and intraoperative navigation in cranio-maxillofacial surgery. Oral Maxillofac Surg Clin North Am. 2010;22(1):135-156.

14. Hernandez-Alfaro F, Guijarro-Martinez R, Molina-Coral A, Badia-Escriche C. "Surgery first" in bimaxillary orthognathic surgery. J Oral Maxillofac Surg. 2011;69(6):e201207.

15. Uribe F, Janakiraman N, Shafer D, Nanda R. Three-dimensional cone-beam computed tomography-based virtual treatment planning and fabrication of a surgical splint for asymmetric patients: surgery first approach. Am J Orthod Dentofacial Orthop. 2013;144(5):748-758.

16. Janakiraman N, Feinberg M, Vishwanath M, Nalaka Jayaratne YS, Steinbacher DM, Nanda R, Uribe F. Integration of 3-dimensional surgical and orthodontic technologies with orthognathic "surgery-first" approach in the management of unilateral condylar hyperplasia. Am J Orthod Dentofacial Orthop. 2015;148(6):1054-1066.

17. Verstraete MA, Willemot L, Van Onsem S, Stevens C, Arnout N, Victor J. Surgery-first approach using a three-dimensional virtual setup and surgical simulation for skeletal Class III correction. J Biomech. 2016;49(3):484-487.

18. Bland JM, Altman DG. Statistical methods for assessing agreement between two methods of clinical measurement. Lancet. 1986;1(8476):307-310.

19. Donatsky O, Bjorn-Jorgensen J, Holmqvist-Larsen M, Hillerup S. Computerized cephalometric evaluation of orthognathic surgical precision and stability in relation to maxillary superior repositioning combined with mandibular advancement or setback. J Oral Maxillofac Surg. 1997;55(10):1071-1079; discussion 1079-1080.

20. Tng TT, Chan TC, Hagg U, Cooke MS. Validity of cephalometric landmarks. An experimental study on human skulls. Eur J Orthod. 1994;16(2):110-120.

21. Padwa BL, Kaiser MO, Kaban LB. Occlusal cant in the frontal plane as a reflection of facial asymmetry. J Oral Maxillofac Surg. 1997;55(8):811-816; discussion 817.

22. Ong TK, Banks RJ, Hildreth AJ. Surgical accuracy in Le Fort I maxillary osteotomies. Br J Oral Maxillofac Surg. 2001;39(2):96-102.

23. Quevedo LA, Ruiz JV, Quevedo CA. Using a clinical protocol for orthognathic surgery and assessing a 3-dimensional virtual approach: current therapy. J Oral Maxillofac Surg. 2011;69(3):623-637. 
24. Stokbro K, Aagaard E, Torkov P, Bell RB, Thygesen T. Virtual planning in orthognathic surgery. Int J Oral Maxillofac Surg. 2014;43(8):957-965.

25. Lin HH, Lo LJ. Three-dimensional computer-assisted surgical simulation and intraoperative navigation in orthognathic surgery: a literature review. J Formos Med Assoc. 2015;114(4):300-307.

26. Hsu SS, Gateno J, Bell RB, Hirsch DL, Markiewicz MR, Teichgraeber JF, Zhou X, et al. Accuracy of a computeraided surgical simulation protocol for orthognathic surgery: a prospective multicenter study. J Oral Maxillofac Surg. 2013;71(1):128-142.

27. Aboul-Hosn Centenero S, Hernandez-Alfaro F. 3D planning in orthognathic surgery: $\mathrm{CAD} / \mathrm{CAM}$ surgical splints and prediction of the soft and hard tissues results
- our experience in 16 cases. J Craniomaxillofac Surg. 2012;40(2):162-168.

28. De Riu G, Meloni SM, Baj A, Corda A, Soma D, Tullio A. Computer-assisted orthognathic surgery for correction of facial asymmetry: results of a randomised controlled clinical trial. Br J Oral Maxillofac Surg. 2014;52(3):251257.

29. Zhang N, Liu S, Hu Z, Hu J, Zhu S, Li Y. Accuracy of virtual surgical planning in two-jaw orthognathic surgery: comparison of planned and actual results. Oral Surg Oral Med Oral Pathol Oral Radiol. 2016;122(2):143-151.

30. Ko EW, Lin CH, Chen YA, Chen YR. Enhanced surgical outcomes in patients with skeletal Class III facial asymmetry by 3-dimensional surgical simulation. J Oral Maxillofac Surg. 2017. 